\title{
Cost-Utility Analysis of Liraglutide in Type 2 Diabetes Patients in China after Chinese Reformation of Medical Care System
}

\author{
Gaoyu Xie1, Suning Zhao² \\ ${ }^{1}$ School of Mathematics, Beijing Normal University, Beijing, China \\ ${ }^{2}$ Shanghai Medical College, Fudan University, Shanghai, China \\ Email: zhujunmin.2008@163.com
}

How to cite this paper: Xie, G.Y. and Zhao, S.N. (2018) Cost-Utility Analysis of Liraglutide in Type 2 Diabetes Patients in China after Chinese Reformation of Medical Care System. Journal of Biosciences and Medicines, 6, 13-25.

https://doi.org/10.4236/jbm.2018.612002

Received: October 29, 2018

Accepted: December 2, 2018

Published: December 5, 2018

Copyright $\odot 2018$ by authors and Scientific Research Publishing Inc. This work is licensed under the Creative Commons Attribution International License (CC BY 4.0).

http://creativecommons.org/licenses/by/4.0/

\begin{abstract}
Objectives: The cost-utility analysis of Liraglutide is aimed at evaluating whether Liraglutide is cost-effective or not after Chinese reformation on medical insurance. The analysis is based on the results of clinical trial conducted in Asia. Methods: We applied a Markov model to estimate the quality-adjusted life years, medical cost and incidence of diabetes-related complications for patients receiving the Liraglutide as an add-on to the metformin treatment. Baseline characteristics were taken from a China's study while the treatment effect is from an Asian study. The related medical cost and utility score were obtained from a local study in China. Having set 30 years' simulations, the incremental cost-effectiveness ratio was calculated comparing with glimepiride treatment. The ratio would be compared with the willingness to pay for a quality-adjusted-life-year (QALY) which is three times of the GDP per capita in Beijing. Sensitivity analysis was also performed. Result: During a period of 30 years, the base-case analysis which takes discount rate at 3\% shows that Liraglutide $1.8 \mathrm{mg}$ results in an average incremental cost of CNY $82,671.49$, an improvement in 0.12 QALYs and a reduction of incidence of diabetes-related complications comparing to glimepiride. The associated incremental cost-effectiveness ratio is CNY 688,929.08. Conclusion: Long-term project shows that taking Liraglutide as an add-on to the metformin treatment will lead to increasing quality-adjusted life years and reduction of incidence of diabetes-related complications. When the price of Liraglutide is reduced by 43 percent in China's yuan, Liraglutide will be cost-effective in China from the healthcare system perspective taking three times of GDP per capita as our WTP threshold.
\end{abstract}

\section{Keywords}

Liraglutide, Type 2 Diabetes Mellitus, Markov Model, Cost-Effectiveness 
Analysis, Chinese Reformation on Medical Insurance

\section{Introduction}

Type 2 Diabetes Mellitus (T2DM) is a progressive endocrine disease caused by insulin resistance or impaired insulin secretion. According to estimation, the prevalence of T2DM has been increased significantly since 1970s, reaching 9.7\% in 2010-2014 [1]. About 50 million people will suffer from T2DM in 2025, which will bring a heavy burden to our society and health systems [2].

As the reforming of Chinese medical care system, the expanding of medical insurance area has attracted a lot of attention. In 2017, the Ministry of Human Resources and Social Security of the People's Republic of China (now the medical insurance managed by the Ministry of Medical Security of the People's Republic of China) has announced that 36 drugs could be covered by our medical insurance, which means that people could buy these 36 drugs at a lower price [3]. Being one of them, Liraglutide's price has reduced from 771.93 RMB to 410 RMB per $18 \mathrm{mg}$, bringing Chinese T2DM patients a new hope.

Liraglutide was introduced into China in 2011 as a T2DM drug, which is a glucagon-like peptide-1 (GLP-1) receptor agonist. It has 97\% amino acid sequence identity (homology) to human endogenous GLP-1, which accounts for $<20 \%$ of total circulating endogenous GLP-1, but it has an Arg 34 replacing Lys 34 at the N-terminal and a fatty acid chain added to Lys 26 . With such features, Liraglutide could bind to and activate the GLP-1 receptor which results in increasing of intracellular cylic adenosine monophosphate (cAMP) and releasing of insulin [4]. Thus, it could regulate blood sugar level, serving as a T2DM drug.

Recently, some new researches have demonstrated that Liraglutide has other several effects including controlling weight [5], relieving cardiac disease [6], and preventing psychiatric disorders [7]. Obviously, Liraglutide has a great development prospect. However, before our reformation, the majority of patients couldn't afford it. Therefore, it has a huge need to reduce its cost but whether its cost is reasonable enough remains unknown until now. Our research is aiming at discussing the effect of Chinese medical insurance reformation on Liraglutide and T2DM. Because of the lacking of reliable clinical data on Chinese group, we still use NCT00614120 trial conducted by Novo Nordisk [8], but we have used TreeAge software to construct a new model so as to be more suitable for Chinese situation.

\section{Material and Method}

\subsection{Model Description}

We applied a state-transition Markov model [9] [10] using decision analysis software (TreeAge Pro, 2011, Williamstown, MA, USA) to evaluate the cost-effectiveness of Liraglutide in China. The ingredients of the model will be 
subsequently introduced. And all the data were analyzed using TreeAge Pro Suite 2011.

In this model, we distinguish different states of diabetes patients on the basis of complications of the disease. Based on that we divided our model into three states which are diabetes, diabetes complications and death. People in the "Diabetes" states are defined as only suffering from diabetes. Likewise, "Diabetes complications" states are defined as patients suffering from more than one kind of diabetes-related complications. Finally, if patients died from any cause related to diabetes or its complications, they enter intothe "Death" state. We define one year as a cycle length (Figure 1).

And then we simulated 1000 patients whose baseline characteristics and risk factors were based on the Chinese study [11] as our input. Then we let those subjects enroll in the Markov simulations. Based on the cohort criterion of UKPDS, we assume that every patient entering the simulations do not suffer from long-term complications. It is also reasonable that the transition probability between each state is constant in our model. The prevalence of diabetes, diabetes complications and death are derived from the study of UKPDS 34 [12]. While simulating each "patient" behavior in our model, we would allocate the "patients" to different stages based on the transfer rate. Then after several times of recycling, we get a new cohort's distributions and can calculate each cycle's cost, cumulative cost, cycle's effect and cumulative effect during the simulations. When a "patient" dies in one cycle, qualified-adjusted living years (QALYs), cumulative clinical cost can be calculated.

\subsection{Treatment Effect}

Treatment effect of Liraglutide added to metformin was taken from the clinical trial (NCT0061412) which aims at comparing the effect on glycaemia control of Liraglutide. This trial was conducted in Asian countries such as China, South Korea and India and was designed similarly as LEAD-2 trial except for a metformin plus placebo arm. In the 16-week trial, Liraglutide led to significant glycemic control but with less obvious loss in weight. In this trial Liraglutide could make reduction in $\mathrm{HbA} 1 \mathrm{c}$ for 1.0 percent $(0.6 \mathrm{mg}), 1.3$ percent $(1.2 \mathrm{mg})$ and 1.4 percent $(1.8 \mathrm{mg}$ ) comparing to the initial $\mathrm{HbAlc}$ rate. The baseline of $\mathrm{HbAlc}$ was extracted from a clinical trial conducted in China [9]. We applied Clark's

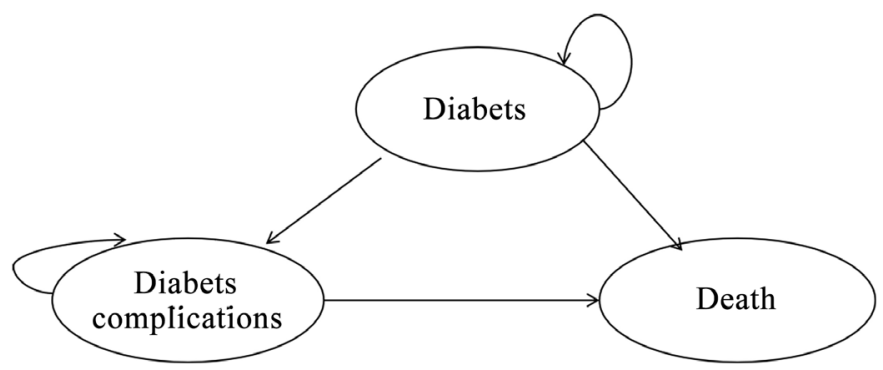

Figure 1. Structure of Markov model. 
study [13] to estimate the related decrease of diabetes complications. By the study of Clark [13] in 1997, which stated that patients with HbA1c between $7 \%$ to $11 \%$, the related decrease of $10 \%$ in $\mathrm{HbA} 1 \mathrm{c}$ could result in $40 \%$ decrease of incidence of complications.

\subsection{Cost and Perspective}

The clinical cost for diabetes patients could be divided into direct clinical cost and indirect clinical cost. The direct clinical cost includes managing diabetes, anti-diabetes treatments and curing diabetes complications. All the direct clinical costs are derived from the clinical trial in China and the acquisition cost of Liraglutide, insulin, glimepiride and metformin were derived from official web sites of Chinese government [14]. For the use of Liraglutide, since none of the treatments nowadays affect the progression loss of beta-cell function, we set the usage of Liraglutide to 5 years in our models. Since adverse events cost takes a minor part of the total cost, we do not include it into our model.

\subsection{Utilities}

Since our main focus is the benefits of the price change of Liraglutide, it is appropriate for us to use quality-adjusted-life-years (QALYs) and incremental cost per QALYs (ICRE) as our principal outcomes. The initial score of our model were taken from a study conducted in Nanjing, China [15]. Based on this study, patients without complications were assumed to have an average EQ-5D scores of 0.863 , patients with more than one complication have an average EQ-5D scores of 0.692. Apparently, people who die have 0 score of EQ-5D.

\subsection{Discount Rate and Time-Horizon}

Both the discount rate of cost and QALYs are 3\% according to the recommendation of World Health Organization (WHO) [16]. By analyzing previous studies of Liraglutide's cost-effectiveness, we set the time horizon to 30 years to explore the cost-effectiveness of Liraglutide in China.

\subsection{Willingness to Pay for a QALY Gained}

Based on the article dealing with the maximum willing to pay (WTP) for a QALY gained in China [17], there still lacks articles discussing the WTP for a QALY towards diabetes in China. So according to the recommendation of the Commission on Macroeconomics and Health of the World Health Organization, the maximum value of one year of healthy life is about three times of the Gross Domestic Production (GDP) per capita.

Since the gross domestic product differs greatly in different part in China, we use the gross domestic product (GDP) per capita in Beijing. In 2017, the gross domestic product (GDP) per capita in Beijing was 128,927 RMB (8826 US\$). Using this figure, we could assume that the maximum WTP for a QALY gained in China might be about 386,781 RMB. 


\subsection{Sensitivity Analysis}

We applied several one-way sensitivity analyses to test the robustness of our primary model. To analyze the uncertainty of the medical cost on different studies, two different methods were applied for sensitivity analysis, which are increasing and decreasing the medical cost by 20 percent respectively. These two methods are used in both medical cost of diabetes and its complications. The impact of discount rate was analyzed by using different discount rates, which are 0 percent and 6 percent. Additionally, to investigate the influence of different time duration towards QALYs and expected cost the time, we changed the horizon from 10 years to 20 years.

\section{Results}

\subsection{Clinical Cost}

The direct medical cost of patients both with and without complications are derived from a study of China [11] and the details of the medical cost are shown in Table 1. Since the medical cost was calculated using US dollar, we apply the exchange rate from US dollar to RMB to be 6.2 in the study. The medical cost mentioned in the study did not include the price of Liraglutide and glimepiride. So we get the price of Liraglutide and glimepiride from the official website of China National Drug Administration and the preferred usage per day is derived from the clinical trial (NCT00614120) which are $1.8 \mathrm{mg}, 1.2 \mathrm{mg}$ per day for Liraglutide and $4 \mathrm{mg}$ per day for glimepiride respectively. Since the price of glimepiride varies in different brands, we use weight-average medical cost for patients with complications and the average price of glimepiride sold in Beijing for the simplicity of our model. Therefore, the medical cost of glimepiride is 1326.15 RMB per year and the medical cost of Liraglutide is $19,865 \mathrm{RMB}(1.8 \mathrm{mg})$ and 9976.67 RMB (1.2 mg).

\subsection{Transition Probability}

Based on the Markovian assumption [9], we assume that the transition probabilities are constant over time. The three key transition probabilities in our model are diabetes to diabetes, diabetes to death, diabetes complications to diabetes complications. All the transitions are one-way and all the people who die remain in the death states. Since diabetes is a chronic disease, all the patients will not return to the diabetes states if once diagnosed with diabetes complications.

The duration of the clinical trial (NCT00614120) that was included in was 16 weeks. Based on the Clark's study [13] and trial conducted in China, we can properly assume that the 16-week trial has the same treatment effect as the one-year treatment. The baseline of the annual transition rate from diabetes transforming to macrovascular complications and microvascular complications can be obtained from the United Kingdom Prospective Diabetes Study (UKPDS) [12] which takes the patient treated with metformin as the object. The Clark's study [13] shows that if the concentration of $\mathrm{HbAlc}$ is between $7 \%$ to $11 \%$, then 
the $10 \%$-decreasing of relative concentration of $\mathrm{HbA} 1 \mathrm{c}$ will result in $40 \%$ reduction in the relative risk of diabetes complications. Since the baseline concentration of HbAlc for patients in China is $7.8 \%$ [11], it is practicable for us to apply it in our study. Though the study is based on the trial in Type I diabetes patients, many experts [18] [19] [20] consider it feasible in Type II diabetes patients. Table 2 shows the detail of how the treatment of Liraglutide affects the concentration of $\mathrm{HbA} 1 \mathrm{c}$ and diabetes complications rate. And Table 3 shows annual incidence of diabetes complications and death events.

Mostly the transition information in the clinical trial is shown in "rate" but not "probability" and so as the UKPDS information in Table 1 . However, in a certain period, we could use rate to estimate the transition probability [21] by using the formula: $P=1-\mathrm{e}^{-r t}$.

The annual state transition probability in group with different dosage of Liraglutide can be obtained from Table 4.

\subsection{Cohort Analysis}

By assuming that the transformation for a fixed number of patients at certain states will comply with certain probability, we could cumulatively calculate the average time in different states to do the cohort analysis. The calculation process is implemented in TreeAge Pro Suite 2011. In the 30 years' simulations for patients treated with $1.2 \mathrm{mg}$ Liraglutide per day, there are $70.9 \%$ of patients remain at diabetes states, while $20.9 \%$ of patients transform into diabetes complications states and $8.1 \%$ of patients die during in this 30 -year simulations. Comparing with the previous treatment, only $18.7 \%$ of patients transfer into diabetes complications and $6.5 \%$ of patients die during the simulations.

\subsection{Base Case Analysis}

The effects and costs of the two treatments during 30 years of follow-up are summarized in Table 5. At the end of the simulation, the $1.2 \mathrm{mg}$ dosage group results in no incremental gain of QALYs comparing to the without Liraglutide group. Moreover, the $1.2 \mathrm{mg}$ dosage group cost 55,351.69 RMB more, so it is not cost-effective for this kind of treatment. While for the $1.8 \mathrm{mg}$ dosage treatment result in an incremental cost of $82,671.49$, and an incremental gain of 0.12 QALYs. The related ICER is 6,888,929.08 which is greater than the WTP for a QALY gain. So we could not conclude that liraglutide as cost-effective if we take glimepiride as comparator.

\subsection{Sensitivity Analysis}

Without changing the cost and discount rate, both the discount rate of $0 \%$ and $6 \%$ only led to 0.06 QALYs change from the base case, and the associated ICER is $403 \mathrm{~K} \mathrm{RMB} / \mathrm{QALY}$ and $1104 \mathrm{~K} \mathrm{RMB} / \mathrm{QALY}$ respectively. When the simulation period changing from 10 years to 20 years, ICER change from $1379 \mathrm{~K}$ RMB/QALY to $4148 \mathrm{~K}$ RMB/QALY. When changing the diabetes' complications 
medical cost from 7013.448 RMB to 10,520.172 RMB, analysis results in ICERs ranging from $1 \mathrm{~K} \mathrm{RMB/QALY}$ to $402 \mathrm{~K} \mathrm{RMB} / \mathrm{QALY}$. While likewise for the diabetes' medical cost change, it results in ICERs ranging from $371 \mathrm{~K} \mathrm{RMB/QALY}$ to $407 \mathrm{~K} \mathrm{RMB/QALY}$. So ICER is more sensitive to discount rate, time duration and the diabetes-related complications cost. The details of sensitivity analysis result are summarized in Table 6.

Table 1. Medical cost of patients with and without complications (in US dollar).

\begin{tabular}{ccc}
\hline & Mean (SD) & Median (IQR) \\
\hline $\begin{array}{l}\text { No complications } \\
\text { Microvascular } \\
\text { complications }\end{array}$ & $1414.0(1388.5)$ & $1086.1(485.4-1849.5)$ \\
$\begin{array}{l}\text { Macrovascular } \\
\text { complications }\end{array}$ & $2470.2(4396.0)$ & $1545.2(841.1,2431.0)$ \\
Micro- \& Macro vascular \\
complications
\end{tabular}

SD: standard deviation. IQR: interquartile range.

Table 2. Relative change from baseline in HbAlc and diabetes complications.

\begin{tabular}{|c|c|c|c|}
\hline & $\begin{array}{l}\text { HbAlc reduction } \\
\text { from baseline (\%) }\end{array}$ & $\begin{array}{c}\text { HbAlc relative } \\
\text { reduction } \\
\text { from baseline }(\%)\end{array}$ & $\begin{array}{c}\text { Change in related } \\
\text { prevalence of } \\
\text { complications }\end{array}$ \\
\hline \multicolumn{4}{|l|}{ Treatments } \\
\hline $\begin{array}{c}1.2 \mathrm{mg} \text { Lirag + } \\
\text { Glim + Met }\end{array}$ & $-1.3 \%$ & $-16.67 \%$ & $-66.67 \%$ \\
\hline $\begin{array}{c}1.8 \mathrm{mg} \text { Lirag + } \\
\text { Glim + Met }\end{array}$ & $-1.4 \%$ & $-17.95 \%$ & $-71.79 \%$ \\
\hline Glim $4 \mathrm{~g}+$ Met & $-1.3 \%$ & $-16.67 \%$ & $-66.67 \%$ \\
\hline
\end{tabular}

Lirag: Lilraglutide; Glime: Glimpepirid; Met: Metformin.

Table 3. Baseline characteristic and treatment effect of different dosage.

\begin{tabular}{cccc}
\hline baseline & $\begin{array}{c}1.2 \mathrm{mg} \\
\text { Lirag + Glim }\end{array}$ & $\begin{array}{c}1.8 \mathrm{mg} \\
\text { Lirag + Glim }\end{array}$ \\
\hline $\begin{array}{c}\text { Mircrovascular } \\
\text { complications }\end{array}$ & 0.0210 & 0.0069 & 0.0059 \\
$\begin{array}{c}\text { Macrovascular } \\
\text { complications } \\
\begin{array}{c}\text { Non-vascular } \\
\text { disease mortality }\end{array}\end{array}$ & 0.0067 & 0.0022 & 0.0019 \\
death & 0.0068 & 0.0023 & 0.0019 \\
\hline
\end{tabular}

Lirag: Lilraglutide; Glime: Glimpepirid; Met: Metformin. 
Table 4. Annual state transition probability in group with different dosage of Liraglutide.

\begin{tabular}{ccccc}
\hline & & $\begin{array}{c}\text { Values for } \\
1.2 \mathbf{~ m g}\end{array}$ & $\begin{array}{c}\text { Value for } \\
1.8 \mathrm{mg}\end{array}$ & $\begin{array}{c}\text { Value for } \\
\text { Glime group }\end{array}$ \\
\hline $\begin{array}{c}\text { Parameter } \\
P_{1}\end{array}$ & Event & & & \\
$P_{2}$ & State I toState I or II & 0.9977 & 0.9981 & 0.9977 \\
$P_{3}$ & State I to State III & 0.0023 & 0.0019 & 0.0091 \\
$P_{4}$ & State II to State II & 0.9932 & 0.9943 & 0.0023 \\
$P_{5}$ & State II to State III & 0.0068 & 0.0057 & 0.0068 \\
$P_{6}$ & Stay in State I & 0.9909 & 0.9922 & 0.9909 \\
$P_{7}$ & Stay in State II & 0.0091 & 0.0078 & 0.0091 \\
\hline
\end{tabular}

State I: Patients with diabetes state in model; State II: Patients with diabetes complications in model; State III: Patients die in model.

Table 5. Results of cost-utility analyses.

\begin{tabular}{|c|c|c|c|c|c|c|}
\hline Strategy & Cost (RMB) & $\begin{array}{l}\text { Incremental } \\
\text { costs }\end{array}$ & Effectiveness & $\begin{array}{l}\text { Incremental } \\
\text { effectiveness }\end{array}$ & $\mathrm{C} / \mathrm{E}$ & $\begin{array}{l}\text { Incremental } \\
\mathrm{C} / \mathrm{E} \text { (ICER) }\end{array}$ \\
\hline $\begin{array}{c}\text { Glimepiride + } \\
\text { metformin }\end{array}$ & $210,502.91$ & & 16.68 QALY & & $12,620.07$ & \\
\hline $1.2 \mathrm{mg}$ & $265,854.60$ & $55,351.69$ & 16.68 QALY & 0 & $15,938.53$ & 0 \\
\hline $1.8 \mathrm{mg}$ & $293,174.43$ & $82,671.49$ & 16.80 QALY & 0.12 & $17,450.86$ & $40,256.58$ \\
\hline
\end{tabular}

$1.2 \mathrm{mg}$ : patients receive $1.2 \mathrm{mg}$ liraglutide per day; $1.8 \mathrm{mg}$ : patients receive $1.8 \mathrm{mg}$ liraglutide per day; $\mathrm{C} / \mathrm{E}$ : cost/effectiveness.

Table 6. Results of sensitivity analysis.

\begin{tabular}{ccccccc}
\hline & \multicolumn{3}{c}{$1.8 \mathrm{mg}$ Lirag } & & \multicolumn{2}{c}{ Glim } \\
\cline { 2 - 7 } & Cost/RMB & QALYs & Cost/RMB & ICER & QALYs & Cost/RMB \\
\hline Trial 20 & $214,026.96$ & 12.90 & $241,436.06$ & $1,379,344.167$ & 12.84 & $158,675.41$ \\
Trial 10 & $145,333.51$ & 7.49 & $172,951.67$ & $4,148,488.5$ & 7.47 & $89,981.90$ \\
Discount rate 0 & $373,685.54$ & 24.80 & $402,867.79$ & $403,140.23$ & 24.58 & $314,176.94$ \\
Discount rate 6\% & $203,222.49$ & 12.21 & $228,851.81$ & $1,104,957.71$ & 12.14 & $151,504.77$ \\
complications - 20\% & $199,511.61$ & 12.21 & $225,601.41$ & $301,783.86$ & 12.14 & $204,476.54$ \\
Comp cost + 20\% & $206,933.38$ & 12.21 & $232,102.21$ & 1275.42 & 12.14 & $216,529.29$ \\
Diabetes cost $-\mathbf{2 0 \%}$ & $180,400.75$ & 12.21 & $208,899.32$ & $407,122.43$ & 12.14 & $179,614.29$ \\
Diabetes cost + 20\% & $226,044.50$ & 12.21 & $252,054.96$ & $371,578.00$ & 12.14 & $241,391.54$ \\
\hline
\end{tabular}

Lirag: liraglutide; Glim: glimepiride; Comp: Complications;

\section{Discussion}

The process of Chinese health care system reformation has already drawn a lot of attention all over the world. In the reformation on health care insurance, Chinese government has done a series of negotiations with pharmaceutical 
companies on cutting customs in order to relief the economics burden of Chinese patients. During recent years, the prices of nearly 50 kinds of drugs have already been cut down to be affordable, which has brought good news to Chinese people in many aspects.

When Liraglutide was firstly introduced to the market in China, the majority of patients couldn't afford due to its expensive cost. However, in 2017, its price has been cutting down about $45 \%$ to 410 yuan per $18 \mathrm{mg}$ and has been included into Chinese medical insurance. In 2012, some researchers have already done some researches using cost-utility analysis on the Liraglutide in Chinese market [22], but due to the change in China these years, the results are not suitable for the situation now. Therefore, we did new researches on the reasonability of the newest price of Liraglutide.

The result of our model shows that Liraglutide treatment might not be cost-effective if we choose glimepiride treatment as comparator. The ICER value obtained from our cost-utility analysis shows that the ICER of Liraglutide exceeds too much from the threshold of $386,781 \mathrm{RMB} / \mathrm{QALY}$, which is the calculated from the three times of GDP [17]. From the clinical trial we could see that the reduction of $\mathrm{HbAlc}$ from baseline shares less disparity between the treatment with $1.8 \mathrm{mg}$ dosage of Liraglutide and glimepiride control group. Even the price of Liraglutide decrease greatly due to the Chinese reformation of medical care system, it is still not effective for the Chinese patients to add Liraglutide in their treatment. In order to achieve the cost-effective goal, the price of Liraglutide should be reduced to $231.3 \mathrm{RMB}$, which is almost a 50 percent reduction from the set price if the government and the company permitted. Our sensitivity analysis shows that apart from time duration and discount rate, the medical cost of diabetes patients is a major factor underlying the ICER outcome.

While comparing to the previous study on Liraglutide in China [22], a great difference in ICER occurs. The difference of the result from our modeling could be explained in several aspects. Firstly, the initial utility score used in our model was derived from a study conducted in Nanjing, China in 2008, which means that the profile of participants in this study are not identical to the clinical trial we refer to. This kind of gap between participants might be result in overstating or understating the utility outcome of our model. Secondly, for the incoherence of participant between the clinical trial and the baseline characteristics, the QALYs and cumulative medical cost might not well reflect the fact. Moreover, the annual incidence of diabetes complications was derived from UKPDS 34 [12] which conducted in 1998 has a period lapse of 20 years. It is likely for the incidence to change during 20 years, however, there is no later study on the incidence of diabetes complications, UKPDS is the preferential one. Thirdly, comparing to the 2012 study [22], the medical cost used in that model is derived from the UKPDS Outcome Model [23] [24] which was developed based on the data from patients with type 2 diabetes in UK. The difference in the economic development and baseline characteristics could lead to difference in the results. Last, the clinical trial we used in this study contained patients from other coun- 
tries such as South Korea and India because until now we still lack related trial conducted only in China, in that case the drug effect might be incoherent to some extent.

There are some limitations in our study. Firstly, the transition probability between each states differ from age and duration of diabetes. However, because of the lack of related data in China, patients in different ages share the same transition probability and possibility of death, which might cause inaccuracies in our model. Secondly, our study was based on a 16-week-study, which is a relatively short duration. Type 2 diabetes is a chronic disease, even in our model we pre-assume that this 16 weeks' study share the same effect as a one-year treatment, the ongoing glycemic control over time is still not quite accurate. Secondly, we adopt 3 times GDP per capita in Beijing as WTP threshold. Even the GDP per capita varies greatly in different areas of China and the prevalence of diabetes increases rapidly, especially in big cities, the generality of this method still need to be considered. Finally, WTP is highly associated with the physical and psychological effect of disease [10] but until now there still lack of certain study on the WTP applying to diabetes in China. Different WTP setting might cause a difference in our study.

According to the annual report of Novo Nordisk [25], in 2017 when Liraglutide officially cut prices in the Chinese market, its products accounted for $10 \%$ of the sales in the Chinese market, with an annual growth rate of $6 \%$, exceeding Novo Nordisk's total revenue growth rate $(2.3 \%)$. Liraglutide has always been a star product of Novo Nordisk. In 2017, the global sales of Liraglutide reached US $\$ 23.173$ billion, a year-on-year increase of $16 \%$. However, we have not obtained specific data in the Chinese market, and since Liraglutide only officially cut prices for half a year, the market popularity may still be low. Therefore, we could not know the impact of the price reduction.

In the Chinese market, we used the example of Iressa (gefitinib) which entered the medical insurance in 2016 as a reference to the Liraglutide. After a sharp price cut, it could be seen that in the first quarter of 2018, Iressa sales reached 849,000 boxes, comparing to 2016 with a 678,000 boxes growth rate, and the quarter-on-quarter growth was as high as $22.2 \%$. Although the price cut exceeded 50\%, in terms of sales, the sales in the first quarter of 2018 was 183 million yuan, which was more than doubled before the price cut. Although the price of it has decreased, there was an increase in sales.

Although this is also due to the successful market strategy of Iressa, we can assume this on Liraglutide. The population of type 2 diabetes in China has reached 120 million. With the increase in the number of diagnosed patients, the demand for diabetes drugs will be more extensive. Liraglutide, a GLP-1 receptor agonist, has been confirmed to have weight loss. It could also regulate cardiovascular function and has anti-dementia effect. The market space of Liraglutide is huge.

According to Novo Nordisk's first quarter earnings conference call in 2018 
[26], after Liraglutide was included in the National Health Insurance Directory last year, the drug sales increased by $53 \%$ in RMB. Based on these circumstances, we think that drugs with such a large market size and market reputation will not affect the company even after the price cut by medical insurance, but increase sales volume by increasing the number of sales. Chinese GLP-1 market accounts for a very small proportion of the total diabetes market, only about $1 \%$, and there is still much room for growth compared to $10 \%-12 \%$ in the European market.

Although Novo Nordisk can still make profits due to the price reduction of drugs at this stage, according to our research results, for the current GDP of the Chinese capital Beijing, to achieve the best economic benefits, the price of Liraglutide must be 231.3 yuan. Moreover, because the GDP of the Chinese capital has a large gap with the national average GDP, in fact, to spread to the whole country, it needs to be sold at a lower price. The current sales of Liraglutide may come from propaganda and other reasons, but from the patient's point of view, prices still need to be lowered. The generic drug of Liraglutide is already under development and hopes to bring tangible gospel to patients in the future.

\section{Conclusion}

Although the Chinese government has made a lot of efforts to reduce the price of imported drugs, in fact, the price of Liraglutide still does not reach the cost-effective price. Because we use GDP as a reference for patients' willing to purchasing in the calculation, this may also be due to the low level of China's GDP. However, there are still many people with type 2 diabetes in China. To truly extend the lifespan of patients, it is still necessary to encourage drug research and development so that patients can really afford the drug price.

\section{Conflicts of Interest}

The authors declare no conflicts of interest regarding the publication of this paper.

\section{References}

[1] Yang, L., Shao, J., Bian, Y., Wu, H., Shi, L., Zeng, L., et al. (2016) Prevalence of Type 2 Diabetes Mellitus among Inland Residents in China (2000-2014): A Meta-Analysis. Journal of Diabetes Investigation, 7, 845-852. https://doi.org/10.1111/jdi.12514

[2] Wang, W., Mcgreevey, W.P., Fu, C., Zhan, S., Luan, R., Chen, W., et al. (2009) Type 2 Diabetes Mellitus in China: A Preventable Economic Burden. American Journal of Managed Care, 15, 593-601.

[3] Ministry of Human Resources and Social Security of the People's Republic of China. (2017) Notice of the Ministry of Human Resources and Social Security on the Inclusion of 36 Drugs in the Category B of the National Basic Medical Insurance, Work Injury Insurance and Maternity Insurance Drug List. http://www.mohrss.gov.cn/SYrlzyhshbzb/shehuibaozhang/zcwj/yiliao/201707/t2017 $\underline{0718 \_274153 . h t m l}$ 
[4] Perry, C.M. (2011) Liraglutide: A Review of its use in the Management of Type 2 Diabetes Mellitus. Drugs, 71, 2347-2373. https://doi.org/10.2165/11208110-000000000-00000

[5] DTB (2017) Liraglutide for Weight Management. BMJ, 55, 78-81.

[6] Takeyuki, H., Yuko, A., Masatsuna, M., Imai, K., Iguchi, D. and Furuta, S. (2018) Liraglutide Relieves Cardiac Dilated Function than DPP-4 Inhibitors. European Journal of Clinical Investigation, 48, e13007. https://doi.org/10.1111/eci.13007

[7] Camkurt, M.A., Lavagnino, L., Zhang, X.Y. and Teixeira, A.L. (2018) Liraglutide for Psychiatric Disorders: Clinical Evidence and Challenges. Hormone Molecular Biology \& Clinical Investigation. Published Online.

[8] Novo Nordisk, A.S. (2018) Effect of Liraglutide or Glimepiride Added to Metformin on Blood Control in Subject with Type 2 Diabetes.

https://www.clinicaltrials.gov/ct2/show/NCT00614120?recrs=e\&rslt=With\&cond=li raglutide\&cntry $=\mathrm{CN} \& \mathrm{rank}=1$

[9] Briggs, A. and Sculpher, M. (1998) An Introduction to Markov Modelling for Economic Evaluation. Pharmacoeconomics, 13, 397-409.

https://doi.org/10.2165/00019053-199813040-00003

[10] Xie, X. and Vondeling, H. (2010) Cost-Utility Analysis of Intensive Blood Glucose Control with Metformin versus Usual Care in Overweight Type 2 Diabetes Mellitus Patients in Beijing, P.R. China. Value in Health, 11, S23-S32.

https://doi.org/10.1111/j.1524-4733.2008.00363.x

[11] Li, X., Xu, Z., Ji, L., Guo, L., Feng, K., Xu, Y., et al. (2018) Direct Medical Costs for Patients with Type 2 Diabetes in 16 Tertiary Hospitals in Urban China: A Multicenter Prospective Cohort Study. Journal of Diabetes Investigation, 2040-1116. https://doi.org/10.1111/jdi.12905

[12] Listed, N. (1998) Effect of Intensive Blood-Glucose Control with Metformin on Complications in Overweight Patients with Type 2 Diabetes (UKPDS 34). UK Prospective Diabetes Study (UKPDS) Group. Lancet, 352, 854. https://doi.org/10.1016/S0140-6736(98)07037-8

[13] Jr, C.C. (1998) The Burden of Chronic Hyperglycemia. Diabetes Care, 21, C32-C34. https://doi.org/10.2337/diacare.21.3.C32

[14] Beijing Pharmaceutical Centralized Purchasing Service Center (2018) Integrated Management Platform of Beijing Medicine Sunshine Purchase.

[15] Luo, Y.J., Fang, Y. and Ding, X.S. (2009) The Quality of Life of Patients with Rype 2 Diabetes Measured by EQ-5D and Its Influential Factors Analysis. China Journal of Pharmaceutical Economics, No. 2, 12-18.

[16] Commission on Macroeconomics and Health of WHO (2018) Investing in Health: A Summary of the Findings of the Commission on Macroeconomics and Health. http://www.who.int/macrohealth/infocentre/advocacy/en/investinginhealth0205200 3.pdf?ua $=1$

[17] Zhao, F.L., Yue, M., Yang, H., Wang, T., Wu, J.H. and Li, S.C. (2011) Willingness to Pay per Quality-Adjusted Life Year: Is One Threshold Enough for Decision-Making? Results from a Study in Patients with Chronic Prostatitis. Medical Care, 49, 267-272. https://doi.org/10.1097/MLR.0b013e31820192cd

[18] Eastman, R.C., Javitt, J.C., Herman, W.H., Dasbach, E.J., Zbrozek, A.S., Dong, F., et al. (1997) Model of Complications of NIDDM: I. Model Construction and Assumptions. Diabetes Care, 20, 725-734. https://doi.org/10.2337/diacare.20.5.725

[19] Eastman, R.C., Javitt, J.C., Herman, W.H., Dasbach, E.J., Copley-Merriman, C., 
Maier, W., et al. (1997) Model of Complications of NIDDM. II. Analysis of the Health Benefits and Cost-Effectiveness of Treating NIDDM with the Goal of Normoglycemia. Diabetes Care, 20, 735-744. https://doi.org/10.2337/diacare.20.5.725

[20] Group, C.E.S. (1998) The Cost-Effectiveness of Screening for Type 2 Diabetes. $J A M A, 280,1757-1763$. https://doi.org/10.1001/jama.280.20.1757

[21] Petitti, D. (2000) Meta-Analysis Decision Analysis and Cost-Effectiveness Analysis. 2nd Edition, Oxford University Press, New York, 141-168.

[22] Gao, L., Zhao, F.L. and Li, S.C. (2012) Cost-Utility Analysis of Liraglutide versus Glimepiride as Add-On to Metformin in Type 2 Diabetes Patients in China. International Journal of Technology Assessment in Health Care, 28, 436-444. https://doi.org/10.1017/S0266462312000608

[23] Clarke, P., Gray, A. and Holman, R. (2002) Estimating Utility Values for Health States of Type 2 Diabetic Patients Using the EQ-5D (UKPDS 62). Medical Decision Making, 22, 340-349. https://doi.org/10.1177/027298902400448902

[24] Clarke, P.M., Gray, A.M., Briggs, A., Farmer, A.J., Fenn, P., Stevens, R.J., et al. (2004) A Model to Estimate the Lifetime Health Outcomes of Patients with Type 2 Diabetes: The United Kingdom Prospective Diabetes Study (UKPDS) Outcomes Model (UKPDS No. 68). Diabetologia, 47, 1747-1759. https://doi.org/10.1007/s00125-004-1527-z

[25] Novo Nordisk (2018) Novo Nordisk Annual Report 2017. https://www.novonordisk.com/annualreport

[26] Novo Nordisk (2018) Novo Nordisk's Operating Profit Decreased by $8 \%$ in Danish Kroner and Increased by 6\% in Local Currencies in the First Three Months of 2018. https://www.novonordisk.com/content/Denmark/HQ/www-novonordisk-com/en_ $\mathrm{gb} /$ home/media/news-details.2189261.html 